\title{
Assessment and optimization of electroporation- assisted tumoral nanoparticle uptake in a nude mouse model of pancreatic ductal adenocarcinoma
}

\author{
This article was published in the following Dove Press journal: \\ International Journal of Nanomedicine \\ I September 2014 \\ Number of times this article has been viewed
}

\author{
Derek Lamont West ${ }^{1,2}$ \\ Sarah B White ${ }^{3}$ \\ Zhouli Zhang ${ }^{4}$ \\ Andrew C Larson ${ }^{4}$ \\ Reed A Omary ${ }^{5}$ \\ 'Department of Diagnostic and \\ Interventional Radiology, ${ }^{2}$ Department \\ of Bioengineering and Nanomedicine, \\ University of Texas Health Sciences \\ Center at Houston, Houston, TX, \\ ${ }^{3}$ Department of Radiology, Medical \\ College of Wisconsin, Milwaukee, \\ WI; ${ }^{4}$ Department of Radiology, \\ Northwestern University, Chicago, IL; \\ ${ }^{5}$ Department of Radiology, Vanderbilt \\ University, Nashville, TN, USA
}

\begin{abstract}
Pancreatic ductal adenocarcinoma (PDAC) is a particularly lethal form of cancer. In 2012, the incidence of PDAC was 43,920. Five-year survival for patients with PDAC is around $6 \%$, regardless of staging, making PDAC one of the deadliest forms of cancer. One reason for this dismal prognosis is chemoresistance to the current first-line therapy, gemcitabine. There are multiple factors that contribute to the chemoresistance observed in pancreatic cancer. Among them, desmoplasia has been increasingly seen as a significant contributor to chemoresistance. To overcome desmoplastic chemoresistance, several novel methods of treatment have been developed. Electroporation is one such novel treatment. High electrical fields are applied to cells to create pores that increase cell permeability. It has been previously demonstrated that electroporation enhances the therapeutic efficacy of anticancer drugs in pancreatic tumor models. Nanoparticle-based drug delivery systems constitute a second novel method to overcome desmoplastic chemoresistance. Due to their intrinsic design advantages, nanoparticles have been shown to increase the effectiveness of chemotherapeutic agents, while further reducing or even eliminating side effects. To date, there have been no studies evaluating the cumulative effect of combining both nanoparticle and electroporation strategies to overcome chemoresistance in PDAC. Our preliminary studies assessed the in vitro and in vivo uptake of doxorubicin-loaded iron oxide nanoparticles as a function of electroporation voltage and timing of administration in pancreatic adenocarcinoma cells. Our studies demonstrated that addition of electroporation to administration of nanoparticles significantly increased the amount of intracellular iron oxide nanoparticle uptake by a PANC-1 cell line in an athymic nude mouse model of PDAC. Further, electroporation-assisted nanoparticle uptake could be significantly altered by changing the timing of application of electroporation.
\end{abstract}

Keywords: electroporation, nanoparticles, tumoral uptake, pancreatic adenocarcinoma, in vivo

\section{Introduction}

Pancreatic ductal adenocarcinoma (PDAC) is one of the most lethal forms of cancer, with a 5-year survival of around 6\%, regardless of staging. In 2012, 43,920 new cases of PDAC were diagnosed in the USA. Further, in the same year, there were 37,390 deaths from PDAC. ${ }^{1}$ This high mortality rate is due in part to the advanced stage of the disease at the time of diagnosis, as well as the lack of effective treatments. ${ }^{2}$ Currently, surgical resection is the only curative treatment, but less than $20 \%$ of patients qualify as surgical candidates. ${ }^{3}$ Advanced PDAC, ie, surgically unresectable locally advanced adenocarcinoma or metastatic disease, accounts for approximately $80 \%$ of cases at the time of diagnosis. ${ }^{4}$

Almost all currently accepted treatment protocols for locally advanced or metastatic PDAC involve intravenous chemotherapy, most commonly gemcitabine. Despite this,
Correspondence: Derek Lamont West Department of Diagnostic and Interventional Radiology, Department of Bioengineering and Nanomedicine, University of Texas Health Sciences Center at Houston, 188I East Road, SCRB-3 6.4638, Houston, TX 77030, USA Tel +l 7|34865428

Email derek.I.west@uth.tmc.edu 
chemoresistance of PDAC to gemcitabine has been widely reported, leading to treatment failure and ultimately death. ${ }^{5}$ While not completely understood, several mechanisms of chemoresistance have been suggested. Among them, desmoplasia has been increasingly seen as a significant contributor to chemoresistance in PDAC. ${ }^{6}$ Desmoplasia is a dense stromal reaction seen in many forms of cancer, but is particularly intense in PDAC. The desmoplastic tissue contains extracellular matrix proteins, including collagen, pancreatic stellate cells, and immune cells, which are all postulated to aid in the growth of PDAC cells. By elevated interstitial fluid pressures and intrinsic structural characteristics, desmoplasia decreases penetration of molecules, such as chemotherapeutic agents, through the tissue. ${ }^{7}$ In PDAC, the hedgehog signaling pathway is critical in the development of desmoplasia. In 2009, Tuveson et al demonstrated that by inhibiting the hedgehog pathway in pancreatic mouse models, the activity of gemcitabine was significantly enhanced. ${ }^{8}$ This finding further demonstrates the role of desmoplasia in PDAC chemoresistance.

In recent years, there has been increased attention paid to chemoresistance as a potential target in the treatment of PDAC. From this attention, electroporation and nanotechnology have emerged as two novel methods developed to reduce PDAC chemoresistance.

Electroporation is the transient application of high electrical fields to cells, creating pores that increase cell permeability. In the past two decades, the mechanism and practical applications of electroporation have been widely investigated, particularly as a means of introducing a range of drugs, DNA, antibodies, and plasmids into cells. ${ }^{9}$ In PDAC, previous studies have demonstrated that electroporation enhances the therapeutic efficacy of anticancer drugs in pancreatic tumor models. ${ }^{10}$

Nanoparticle therapeutics are typically particles comprised of therapeutic entities, such as small-molecule drugs, peptides, proteins, or nucleic acids, and components that assemble with these therapeutic entities, such as lipids and polymers, to form nanoparticles. ${ }^{11}$ Nanoparticle designs combine a therapeutic entity, such as gemcitabine, with specific surface properties, such as polyethylene glycol or other targeting ligands, allowing them to overcome many forms of chemoresistance. Further, nanoparticles are multifunctional, allowing for direct visualization of drug delivery with imaging modalities such as magnetic resonance imaging. ${ }^{11}$ To that end, there have been numerous nanoparticle-based gemcitabine strategies that overcome PDAC chemoresistance. ${ }^{12-18}$
While many studies have separately investigated the efficacy of electroporation or nanoparticle therapeutics in the treatment of PDAC, there have been no studies evaluating the combined efficacy of these strategies. This study assessed and optimized the uptake efficacy of iron oxide nanoparticles in combination with electroporation in PDAC.

\section{Materials and methods}

All experiments were approved by our institutional animal care and use committee at Northwestern University. The study was divided into two parts. The in vitro part of the study evaluated the effects of voltage levels on intracellular uptake of nanoparticles and cell viability. The second part evaluated the effects of electroporation on uptake of iron oxide nanoparticles and the effects of timing of administration of electroporation in relation to administration of the nanoparticles.

\section{In vitro study}

Eighteen $0.5 \mathrm{~cm}$ cuvette $(5 \mathrm{~mm})$ wells containing 2 million PANC-1 cells per well were incubated. The plated cells were divided into control and experimental groups. Nine control wells were incubated in $2 \mathrm{~mL}$ of Dulbecco's Modified Eagle's Medium (DMEM), and nine experimental wells were incubated in a solution of DMEM with $100 \mathrm{nM} / \mathrm{mL}$ of iron oxide and 10\% doxorubicin (Ocean NanoTech LLC, Springdale, AR, USA). The wells were incubated at $37^{\circ} \mathrm{C}$ in $5 \% \mathrm{CO}_{2}$ for 24 hours.

On day 2, after 24 hours, the cells were washed twice in sterile $1 \times$ phosphate-buffered saline. Next, $1 \mathrm{~mL}$ of the designated DMEM was added to the wells. Control cells were given $1 \mathrm{~mL}$ of DMEM without nanoparticles, and the experimental cells were given DMEM with $100 \mathrm{nM} / \mathrm{mL}$ of iron oxide and $10 \%$ doxorubicin. Electroporation of each well was performed, at voltages ranging from 0 to $1,500 \mathrm{~V}$, using an adherent cell electrode with a $0.5 \mathrm{~cm}$ gap (BTX VCM 830; Harvard Apparatus Ltd, Edenbridge, UK). The electroporation protocol is outlined in Table 1. After each electroporation, the electrodes were wiped off with $70 \%$ ethanol. After electroporation, $1 \mathrm{~mL}$ of DMEM was added to each well and incubated at $37^{\circ} \mathrm{C}$ in $5 \% \mathrm{CO}_{2}$ for 24 hours.

On day 3 , the wells were rinsed twice in sterile $1 \times$ phosphate-buffered saline. The cells were then trypsinized with $0.5 \mathrm{~mL}$ of $0.25 \%$ trypsin for 5 minutes, resuspended in $1 \mathrm{~mL}$ of DMEM, and transferred to $1.5 \mathrm{~mL}$ tubes. The tubes were centrifuged at 1,250 rpm for 5 minutes. The supernatant was aspirated and the samples were divided into cell viability and inductively coupled plasma mass spectrometry (ICP-MS) 
Table I Electroporator settings for in vivo and in vitro electroporation

Electroporation settings

Frequency I Hz

Mode LV or HV (fixed)

Voltage $50-1,500 \mathrm{~V} / \mathrm{cm}$

Pulse length $99 \mu \mathrm{s}$

Eight pulses

Interval $100 \mathrm{~ms}$

Polarity unipolar (fixed)

Abbreviations: LV, low voltage; $\mathrm{HV}$, high voltage.

samples. The cell viability sample cells were resuspended in $0.5 \mathrm{~mL}$ of $1 \times$ phosphate-buffered saline and transferred to flow tubes. Cell viability was immediately performed using a Countess automated cell counter (Invitrogen, Carlsbad, CA, USA) and trypan blue. The ICP-MS samples were resuspended in $0.2 \mathrm{~mL}$ of $70 \%$ nitric acid and then sent for ICP-MS with ${ }^{54} \mathrm{Fe}$ and ${ }^{57} \mathrm{Fe}$. This experiment was repeated six times for a total sample size of $n=6$.

\section{In vivo study}

Using a 30 gauge needle, $10 \times 10^{6}$ PANC- 1 cells in $50 \mu \mathrm{L}$ of phosphate-buffered saline were injected subcutaneously into the bilateral flanks of 8-week-old athymic nu/nu mice. This procedure was performed in the Center for Comparative Medicine Lurie facility according to the guidelines established by the Feinberg School of Medicine and Northwestern Memorial Hospital. The mice were monitored until their tumors reached $1 \mathrm{~cm}$ in diameter.

When the tumors had reached $1 \mathrm{~cm}$, the mice were anesthetized with $2 \%-3 \%$ isoflurane. Under anesthesia, the mice received $400 \mathrm{~V}$ electroporation using pincher electrodes and were injected retro-orbitally with $0.1 \mathrm{~mL}$ of doxorubicin nanoparticles (iron concentration $4 \mathrm{mg} / \mathrm{mL}$ ). The timing of electroporation was varied experimentally for each tumor, from electroporation 12 minutes before to 12 minutes after administration of the nanoparticles. For each time point, three tumors were electroporated. After analysis, three more tumors were treated with electroporation 3 minutes before administration of the nanoparticles, for a total of six tumors at that time treatment point. In addition, six tumors were electroporated without administration of nanoparticles, six tumors received nanoparticles without electroporation, and six tumors did not receive any nanoparticles or electroporation.

After electroporation, all mice were euthanized in a $\mathrm{CO}_{2}$ chamber and bilateral thoracotomy was performed. The tumors were excised from the mouse flanks. A portion of each tumor was placed in formalin. From this sample, hematoxylin and eosin slides and Prussian blue slides were created for each tumor. The remaining portion of each tumor sample was weighed and placed in $70 \%$ nitric acid for $6-8$ hours and subsequently sent for ICP-MS analysis of iron content.

\section{Statistical analysis}

Two-way analysis of variance was performed using Statistical Package for the Social Sciences package version 17 software (SPSS Inc, Chicago, IL, USA) to check whether iron concentrations differed by nanoparticle uptake and voltage levels. Similar techniques were used to examine whether electroporation and its timing were related to iron concentration.

\section{Results \\ Cell viability}

Figure 1 shows the percent change in cell viability plotted against change in field strength. Figure 2 shows cell viability plotted as a function of variation in electroporation voltage. PANC-1 cells incubated with nanoparticles and those incubated without nanoparticles showed a decrease in viability as voltage increased. The rate of decrease in cell viability varied. The greatest decrease in viability was seen as the voltage was increased from $0 \mathrm{~V}$ to $400 \mathrm{~V}$. Between $100 \mathrm{~V}$ and $1,500 \mathrm{~V}$, viability decreased slightly less rapidly. Between $400 \mathrm{~V}$ and $1,000 \mathrm{~V}$, viability decreased by the smallest amount as voltage increased.

\section{Pancreatic voltage setting}

Iron uptake by PANC-1 cells incubated with and without iron nanoparticles was plotted against variations in voltage (Figure 2). Across all voltages, there were statistically significant differences between iron concentrations in PANC-1 cells incubated without iron nanoparticles and PANC-1 cells incubated with iron nanoparticles. The differences between

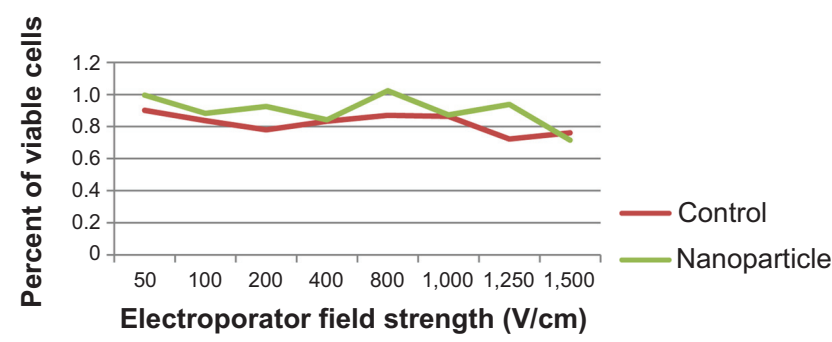

Figure I Percent change in cell viability as a function of field strength. Note: At each field strength, the number of viable cells at that field strength was divided by the number of viable cells at a field strength of $0 \mathrm{~V} / \mathrm{cm}$ to determine the percentage loss of cells after electroporation. 

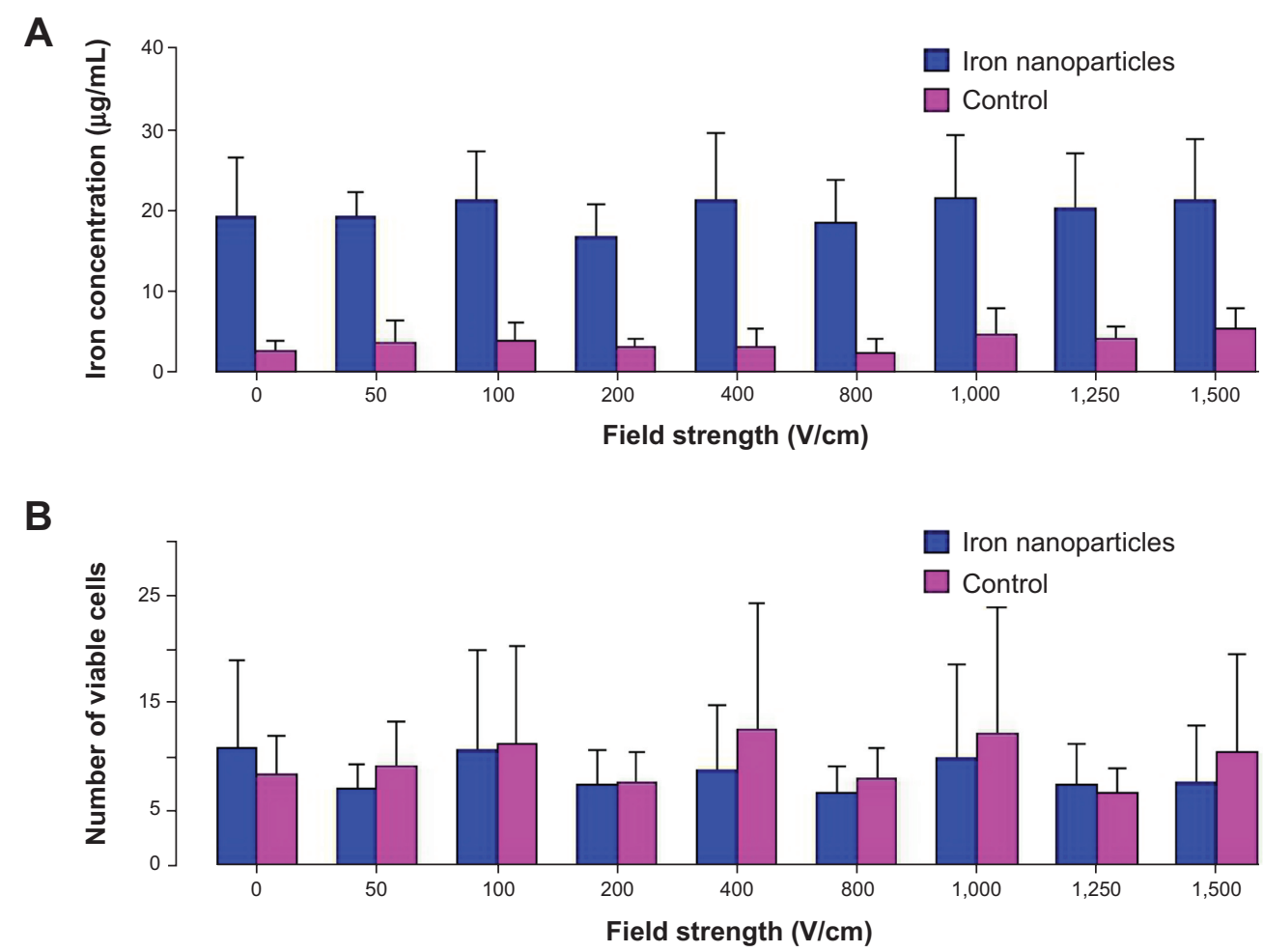

Figure 2 (A) In vitro iron concentration in PANC-I cells and (B) number of viable cells (per 100,000 cells) as a function of electroporation field strength.

the two groups remained constant, regardless of voltage. Both groups showed similar slight increases in iron uptake as voltages increased.

\section{Timing of electroporation}

Figure 3 demonstrates the average iron concentrations in mouse PANC-1 tumors as a function of timing of electroporation in relation to nanoparticle infusion. Similar decreases in iron concentration were seen in the two groups between the -12 minutes and -6 minutes treatment points and between the +2.5 minutes and +12 minutes treatment points. The highest iron concentration was seen at the -3 minutes and +1.5 minutes time points (Table 2, Figure 4 ). Hematoxylin and eosin staining and Prussian blue staining of tumors treated at -3.0 minutes and +1.5 minutes were reviewed (Figures 5A, 5B, 6A, and 6B).

\section{Optimal electroporation timing and control comparisons}

Comparisons of six tumors at the -3.0 minutes time point were compared with iron concentrations in tumors that received no treatment, electroporation only, and nanoparticles only. Statistically significant differences between the -3.0 minutes treatment and the three control arms were seen (Table 3, Figure 3).

\section{Discussion}

The in vitro portion of this study was designed to optimize voltage levels for maximal iron uptake with minimal cell destruction (reversible electroporation). PANC-1 cell cultures incubated with and without iron nanoparticles were electroporated and analyzed for iron content and cell viability. While both groups showed a gradual increase

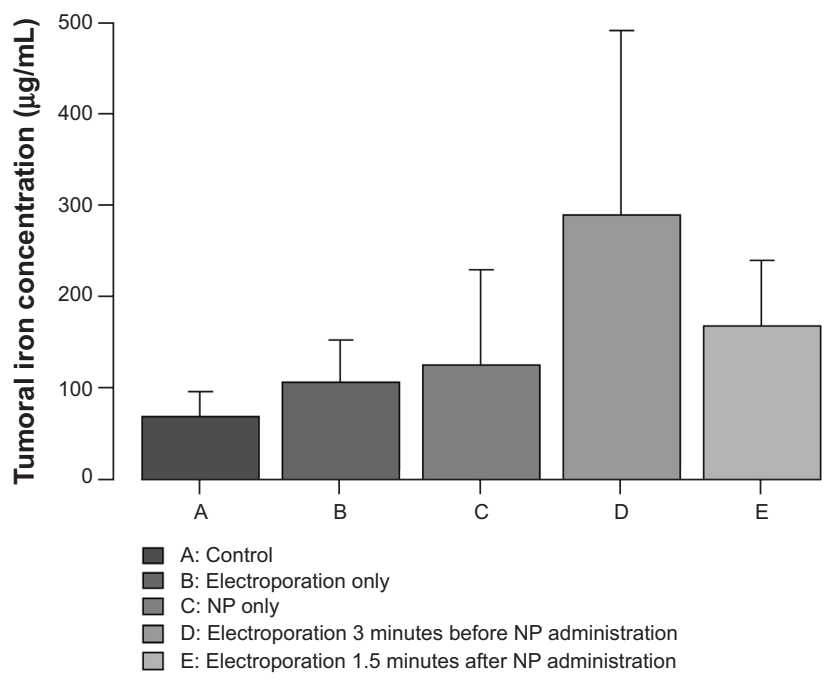

Figure 3 Comparison of intratumoral iron concentration in PANC-I cells in a nude mouse model.

Abbreviation: NP, nanoparticles. 


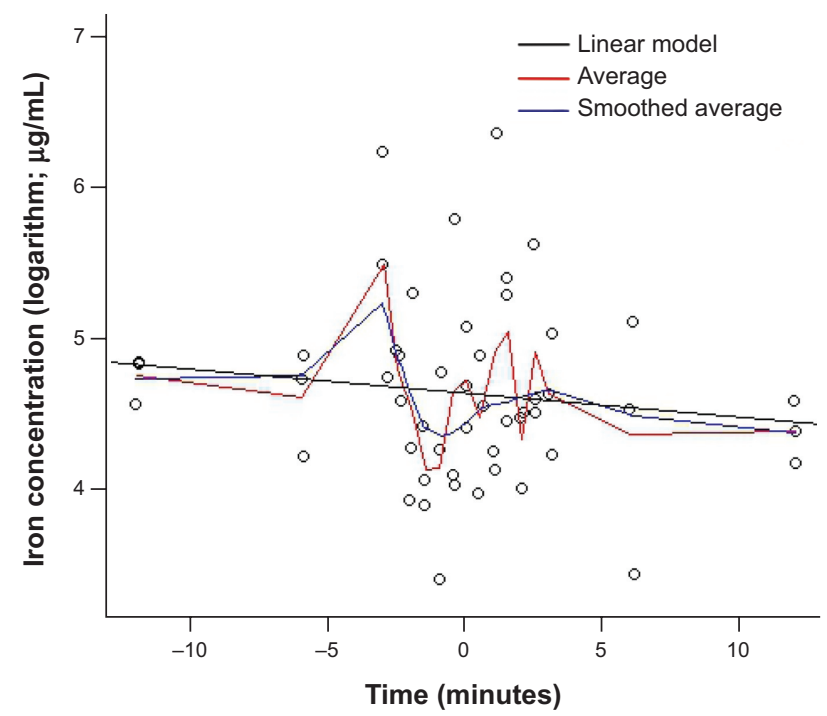

Figure 4 Intratumoral iron concentrations in PANC-I cells in a nude mouse model as a function of timing of electroporation in regard to nanoparticle infusion. Notes: Model fittings from linear regression (in black), simple average (in red), and smoothed average (in blue) are displayed.

in iron content as electroporation voltage was increased, the rate of increase varied, with the steepest increase seen at $400 \mathrm{~V}$. The cells incubated in nanoparticles showed a constantly elevated iron content when compared with the control cells, regardless of voltage. Based on this finding, we postulate that elevated voltages increase the time that the pores remain open, causing increased iron uptake in cells incubated with iron.
The second part of the in vitro study evaluated cell viability as a function of electroporator voltage. PANC-1 cells incubated with iron nanoparticles and PANC-1 cells incubated without nanoparticles both demonstrated decreases in cell viability as voltage increased. However, the rate of decrease varied with cells that were incubated with iron. From $0-400 \mathrm{~V}$ there was a rapid decrease in cell viability rate. From $400 \mathrm{~V}$ to $1,000 \mathrm{~V}$, cells remained relatively stable. Finally, from $1,000 \mathrm{~V}$ to $1,500 \mathrm{~V}$, there was a marked decrease in cell viability, similar to $0-400 \mathrm{~V}$. Cells incubated without iron demonstrated a more consistent linear decrease in viability as the voltage increased.

Several postulates arose from this portion of the study. First, the high rate of cell death in the lowest voltages in the cells incubated with iron may result from the doxorubicin associated with the nanoparticles. The doxorubicin likely subselected death for cells most susceptible to doxorubicin therapy. However, alternative explanations for this high cell death rate at low voltages include variability in the cell threshold for reversible electroporation. Above 1,000 V, cell death likely occurred from depolarization from irreversible electroporation. Given these findings, voltages between $400 \mathrm{~V}$ and $1,000 \mathrm{~V}$ were felt to be optimal for maximal cell viability. This finding supported the threshold phenomena noted in electroporation. Specifically, at a certain defined voltage, reversible electroporation occurs. At a higher defined voltage, irreversible electroporation occurs. Our study

Table 2 Intratumoral PANC-I cellular iron concentrations in the nude mouse as a function of timing of electroporation with regard to administration of nanoparticles

\begin{tabular}{|c|c|c|c|c|}
\hline \multirow{2}{*}{$\begin{array}{l}\text { Timing of electroporation to } \\
\text { nanoparticle administration } \\
\text { (minutes) }\end{array}$} & \multicolumn{4}{|c|}{ Tumor iron concentration $(\mu \mathrm{g} / \mathrm{mL})$} \\
\hline & $\mathbf{I}$ & 2 & 3 & Mean \\
\hline-12.0 & 126.00 & 127.00 & 96.00 & 116.33 \\
\hline-6.0 & 133.00 & 68.00 & 113.00 & 104.67 \\
\hline-3.0 & 115.00 & 242.00 & 510.00 & 289.00 \\
\hline-2.5 & 98.00 & 133.00 & 137.00 & 122.67 \\
\hline-2.0 & 72.00 & 51.00 & 200.00 & 107.67 \\
\hline-1.5 & 58.00 & 49.00 & 83.00 & 63.33 \\
\hline-1.0 & 118.00 & 71.00 & 30.00 & 73.00 \\
\hline-0.5 & 60.00 & 327.00 & 56.00 & 147.67 \\
\hline 0.0 & 161.00 & 82.00 & 108.00 & 117.00 \\
\hline 0.5 & 53.00 & 95.00 & 132.00 & 93.33 \\
\hline 1.0 & 70.00 & 577.00 & 62.00 & 236.33 \\
\hline 1.5 & 221.00 & 197.00 & 86.00 & 168.00 \\
\hline 2.0 & 91.00 & 88.00 & 55.00 & 78.00 \\
\hline 2.5 & 99.00 & 278.00 & 91.00 & 156.00 \\
\hline 3.0 & 69.00 & 154.00 & 102.00 & 108.33 \\
\hline 6.0 & 93.00 & 165.00 & 31.00 & 96.33 \\
\hline 12.0 & 65.00 & 80.00 & 98.00 & 81.00 \\
\hline
\end{tabular}

Note: The experiment was carried out three times $(I-3)$ to obtain the mean. 
A

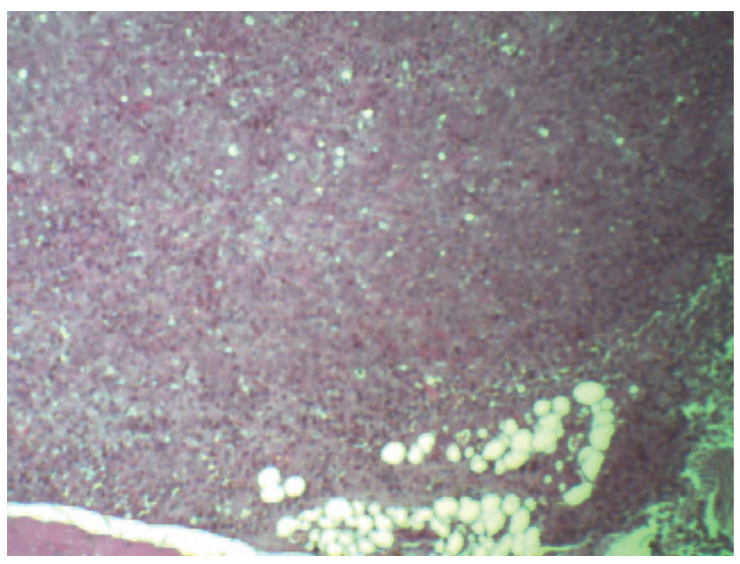

B

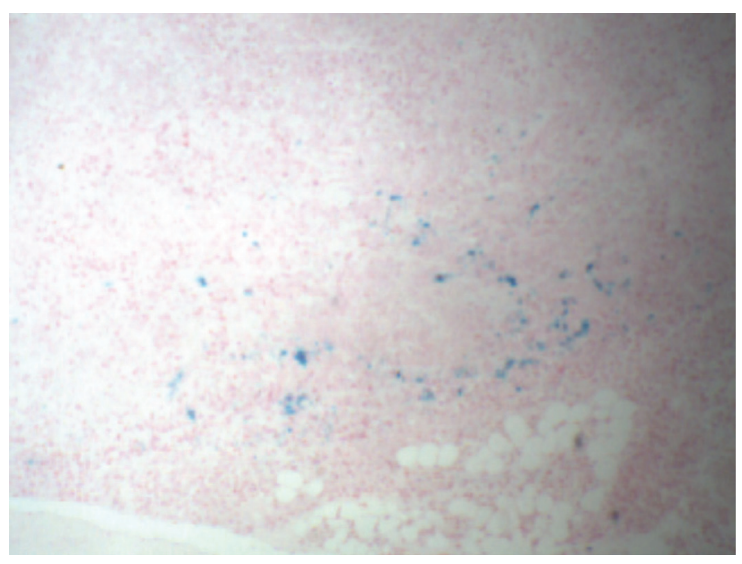

Figure 5 Hematoxylin and eosin staining (A) and Prussian blue staining (B) of PANC-I cells in nude mouse tumor tissue electroporated 3 minutes before administration of iron nanoparticles.

suggests that, for PANC-1 cells, the maximum reversible electroporation voltage is $400 \mathrm{~V}$ and the minimum irreversible electroporation voltage is $1,000 \mathrm{~V}$.

For our study, we chose $400 \mathrm{~V}$ as the voltage for in vivo electroporation. While we demonstrated relative stability of cell viability between $400 \mathrm{~V}$ and $1,000 \mathrm{~V}$, several other studies have shown that cell death from electroporation is directly proportional to voltage settings. This finding supports the threshold phenomena noted in electroporation. Specifically, at a certain defined voltage, reversible electroporation occurs. At a higher defined voltage, irreversible electroporation occurs. Our study suggests that, for PANC-1 cells, the maximum reversible electroporation voltage is $400 \mathrm{~V}$ and the minimum irreversible electroporation voltage is $1,000 \mathrm{~V}$. $^{1}$ From the in vitro iron concentration study, we demonstrated the steepest increase in iron concentration at $400 \mathrm{~V}$. As a result, we elected to use $400 \mathrm{~V}$ as our voltage setting.

Once we selected the voltage, we optimized the timing of electroporation. PANC-1 tumors were randomized to electroporation time intervals ranging from 12 minutes
A

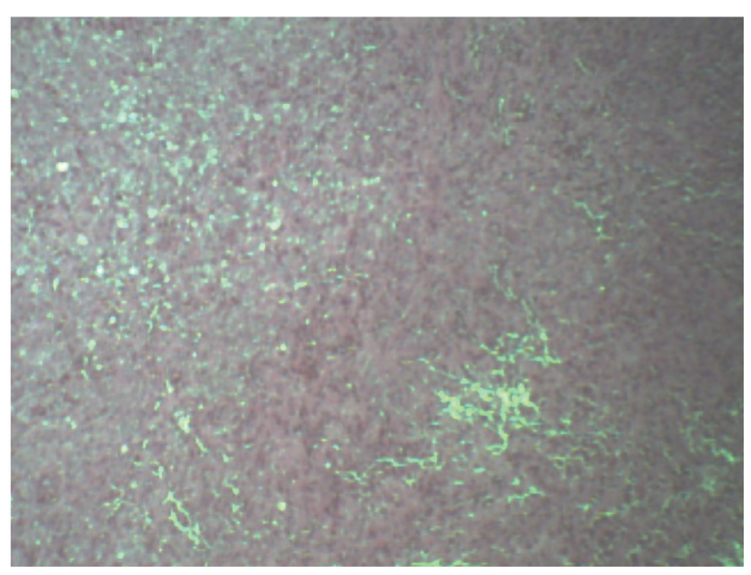

B

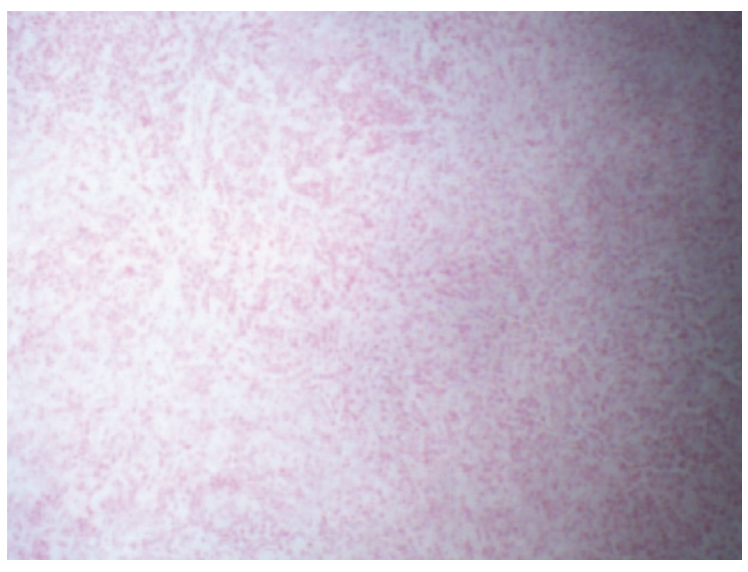

Figure 6 Hematoxylin and eosin staining (A) and Prussian blue staining (B) of a PANC-I nude mouse tumor electroporated 1.5 minutes after administration of iron nanoparticles.

before nanoparticle administration to 12 minutes after nanoparticle administration. Three tumors were assigned to each time point. The average iron content in the three tumors for each time point was plotted graphically against timing of electroporation. Our data suggest two time points that were significant. The first time point was electroporation 3 minutes before nanoparticle administration. Overall, this time point achieved the highest average iron content. The second time point demonstrating significant increase in iron uptake is electroporation 1.5 minutes after nanoparticle administration. Of note, there was a small difference between the electroporation only and nanoparticle only study arms. We did not find this difference to be statistically significant.

Once we obtained the most significant time points, histological examination of each of the six tumors was undertaken. While the hematoxylin and eosin slides of all tumors were relatively similar, Prussian blue staining was markedly different for the tumors. Prussian blue, which stains for iron, demonstrated multiple areas of iron content in the -3 minutes tumors, while the +1.5 minutes tumors demonstrated 
Table 3 Intratumoral iron concentrations

\begin{tabular}{|c|c|c|c|c|c|c|c|}
\hline \multirow[t]{2}{*}{ Study arm } & \multicolumn{7}{|c|}{ Tumoral iron concentration $(\mu \mathrm{g} / \mathrm{mL})$} \\
\hline & I & 2 & 3 & 4 & 5 & 6 & Mean \\
\hline Control & 56.00 & 105.00 & 48.00 & 47.00 & 62.00 & 102.00 & 70.00 \\
\hline Electroporation only & 133.00 & 119.00 & 173.00 & 49.00 & 63.00 & 104.00 & 106.83 \\
\hline NP only & 82.00 & 326.00 & 48.00 & 107.00 & 53.00 & 142.00 & 126.33 \\
\hline $\begin{array}{l}\text { Electroporation } 3 \text { minutes } \\
\text { before NP administration }\end{array}$ & 115.00 & 242.00 & 510.00 & & & & 289.00 \\
\hline $\begin{array}{l}\text { Electroporation } 1.5 \text { minutes } \\
\text { after NP administration }\end{array}$ & 221.00 & 197.00 & 86.00 & & & & 168.00 \\
\hline
\end{tabular}

Note: The experiment was carried out six times $(I-6)$ to obtain the mean.

Abbreviation: NP, nanoparticle.

no visible nanoparticle uptake with Prussian blue. While this may be related to tumor sampling, the lack of iron in the +1.5 minutes tumors was significant. We chose -3.0 minutes as the optimal electroporation time point.

Three more mouse tumors were electroporated at -3.0 minutes, for a total of six tumors. The iron content of the -3.0 minutes tumors was compared with iron nanoparticle only, electroporation only, and control (no treatment) tumors. The tumors electroporated at -3.0 minutes demonstrated a significantly higher iron content than any other group. The highest iron concentration may be achieved when electroporation is performed 3 minutes before nanoparticle administration. However, its standard variation seems relatively large (due to the small samples), so we cannot ignore the possibility that the highest observations are accidentally obtained at 3 minutes before administration of nanoparticles.

We postulate that nanopores may need time for maximal formation. Three minutes seemed to be the optimal time for pore formation in our experiments. Further, nanoparticles are cleared rapidly from the bloodstream by the reticuloendothelial system, limiting the time of tumor exposure to the particles.

There are several important limitations to this study, in that it is very specific in terms of the type of nanoparticles, animal model, and tumor used. While it is likely that electroporation before administration of nanoparticles is optimal, the exact voltage and timing of electroporation with regard to administration would have to be evaluated for each particle type, animal model, and tumor type.

To summarize, in the nude mouse PANC-1 tumor model, we found maximal iron uptake with $400 \mathrm{~V}$ electroporation 3 minutes before administration of iron nanoparticles. To confirm the findings of this study, future research should be conducted to directly visualize nanopore formation and nanoparticle entry. Further studies are also needed to determine the degree of fibrosis, necrosis, and vascularity in each tumor, and to remove these items as confounding factors.

\section{Disclosure}

The authors report no conflicts of interest in this work.

\section{References}

1. Siegel R, Naishadham D, Jemal A. Cancer statistics, 2012. CA Cancer J Clin. 2012;62:10-29.

2. Bond-Smith G, Banga N, Hammond T, Inber C. Pancreatic adenocarcinoma. BMJ. 2012;344:e2476.

3. Li D, Xie K, Wolff R, Abbuzzese JL. Pancreatic cancer. Lancet. 2004;363:1049-1057.

4. Warsame R, Grothey A. Treatment options for advanced pancreatic cancer: a review. Expert Rev Anticancer Ther. 2012;12:1327-1336.

5. Wang Z, Li Y, Ahmad A, et al. Pancreatic cancer: understanding and overcoming chemoresistance. Nat Rev Gastroenterol Hepatol. 2011;8:27-33.

6. Andersson R, Aho U, Nilsson BI, et al. Gemcitabine chemoresistance in pancreatic cancer: molecular mechanisms and potential solutions. Scand J Gastroenterol. 2009;44:782-786.

7. Trendan O, Galmarini C, Patel K, Tannock I. Drug resistance and the solid tumor microenvironment. J Natl Cancer Inst. 2007;99:1441-1154.

8. Olive K, Jacobetz MA, Davidson CJ, et al. Inhibition of hedgehog signaling enhances delivery of chemotherapy in a mouse model of pancreatic cancer. Science. 2009;324:1457-1461.

9. Chen C, Smye SW, Robinson MP, Evans JA. Membrane electroporation theories: a review. Med Biol Eng Comput. 2006;44:5-14.

10. Nanda GS, Sun FX, Hofmann GA, Hoffman RM, Dev SB. Electroporation enhances therapeutic efficacy of anticancer drugs: treatment of human pancreatic tumor in animal model. Anticancer Res. 1998; 18:1361-1366.

11. Davis M, Chen Z, Shin D. Nanoparticle therapeutics: an emerging treatment modality for cancer. Nat Rev Drug Discov. 2008;7:771-782.

12. Réjiba S, Reddy LH, Bigand C, Parmentier C, Couvreur P, Hajri A. Squalenoyl gemcitabine nanomedicine overcomes the low efficacy of gemcitabine therapy in pancreatic cancer. Nanomedicine. 2011;7:841-849.

13. Lieber M, Mazzetta J, Nelson-Rees W, Kaplan M, Todaro G. Establishment of a continuous tumor-cell line (panc-1) from a human carcinoma of the exocrine pancreas. Int J Cancer. 1975;15:741-747.

14. Atkinson AJ, Jr, Huang SM, Jertora JL, Markey SP. Principles of Clinical Pharmacology. 3rd ed. Burlington, MA, USA: Academic Press/ Elsevier; 2012.

15. Elnaggar M, Giovannetti E, Peters GJ. Molecular targets of gemcitabine action: rationale for development of novel drugs and drug combinations. Curr Pharm Des. 2012;18(19):2811-2829.

16. Shimasaki T, Ishigaki $Y$, Nakamura $Y$, et al. Glycogen synthase kinase $3 \beta$ inhibition sensitizes pancreatic cancer cells to gemcitabine. $J$ Gastroenterol. 2012;47:321-333.

17. Herreros-Villanueva M, Hijona E, Cosme A, Bujanda L. Mouse models of pancreatic cancer. World J Gastroenterol. 2012;18:1286-1294.

18. Chen C, Smye SW, Robinson MP, Evans JA. Membrane electroporation theories: a review. Med Biol Eng Comput. 2006;44:5-14. 


\section{Publish your work in this journal}

The International Journal of Nanomedicine is an international, peerreviewed journal focusing on the application of nanotechnology in diagnostics, therapeutics, and drug delivery systems throughout the biomedical field. This journal is indexed on PubMed Central,

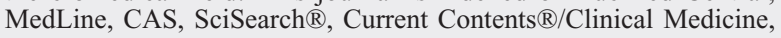

Journal Citation Reports/Science Edition, EMBase, Scopus and the Elsevier Bibliographic databases. The manuscript management system is completely online and includes a very quick and fair peer-review system, which is all easy to use. Visit http://www.dovepress.com/ testimonials.php to read real quotes from published authors.

Submit your manuscript here: http://www.dovepress.com/international-journal-of-nanomedicine-journal 